\title{
A New Method for the Determination of Texture Functions from Incomplete Pole Figures-Comparison with Older Methods
}

\author{
P. VAN HOUTTE
}

Department of Metallurgy and Materials Engineering, Katholieke Universiteit Leuven, de Croylaan 2, B-3030 Leuven, Belgium

(Received December 12, 1983)

Three series expansion methods for texture analysis from incomplete pole figures have been compared. Method I is the presently most used method, method II is a variant of it and method III is entirely new. Computer programs have been written for the three methods.

Incomplete pole figures have then been prepared from known textures of f.c.c. metals. In some cases, artificial experimental errors have been simulated. These pole figures were analyzed by the three computer programs, after which the resulting texture functions were compared to the original texture functions.

No significant differences were found for weak textures. On the other hand, the results of method I are much poorer than those of the two other methods for sharp textures, because it underestimates the normalisation factors.

The best results were obtained by method III.

\section{INTRODUCTION}

The crystallite orientation distribution function (ODF) of a polycrystalline material can be obtained by diffraction experiments. The most complete information is obtained by the transmission electron microscope by measuring the orientation of each crystallite separately. The 
experimental effort however is considerable and so, pole figure measurements on texture goniometers using $x$-ray or neutron diffraction are widely preferred. The pole figures can then be analyzed in various ways: series expansion methods (Bunge, 1965; Roe, 1965) or discrete methods (Williams, 1968; Ruer and Baro, 1977; Imhof, 1982). The oldest methods need complete pole figures but methods which are able to analyze incomplete pole figures have also been developed. Only methods which use the series expansion of the texture functions are considered in this paper (Bunge, 1969; Pospiech and Jura, 1974; Morris, 1975; Van Houtte, 1980; Humbert and Bergmann, 1980; Liang, Xu and Wang, 1981). A new method is proposed and subsequently compared to two of the existing methods. A computer code was written for each of the three methods. Incomplete pole figures were recalculated from known textures. Various types of "experimental errors" were simulated and introduced in some of these pole figures. They were then analyzed by the three methods and the results compared.

\section{CHOICE OF TWO EXISTING METHODS}

\section{Method I}

Most methods try to find a set of $C_{l}^{\mu v}$ coefficients which minimizes the difference between the observed pole figures and the recalculated pole figures (Bunge, 1969):

$$
\Delta=\Sigma w_{i} \int_{B}\left[P_{h_{i}}(y)_{\mathrm{obs}}-P_{h_{i}}(y)_{\mathrm{cal}}\right]^{2} \mathrm{~d} y=\text { Min }
$$

$B$ is the part of the pole figure which has been measured; if the back reflection method was used, this means a range of 0 to $\Phi_{m}$ for $\Phi\left(\Phi_{m}<\pi / 2\right)$ and of 0 to $2 \pi$ for $\beta . w_{i}$ is the weighting factor of pole figure $h_{i}$. The recalculated pole figures depend upon the (unknown) $C_{l}^{\mu \nu}$ coefficients of even rank $1 \uparrow$ (Bunge, 1969):

$$
P_{h_{i}}(y)=\sum_{l=0(2)}^{L} \sum_{v=1}^{N(l)} F_{l}^{v}\left(h_{i}\right) \dot{k}_{l}^{v}(y)
$$

$\dagger$ In order to find the coefficients of odd rank $l$, the non-negativity of the ODF could be used. The discrete methods mentioned above face the same problem under a different mathematical form. 
with

$$
F_{l}^{v}\left(h_{i}\right)=\frac{4 \pi}{2 l+1} \sum_{\mu=1}^{M(l)} C_{l}^{\mu \nu} \dot{k}_{l}^{\mu}\left(h_{i}\right)
$$

$y$ refers to a particular $\Phi-\beta$ direction in the pole figure; $h_{i}$ refers to a particular pole figure (e.g. $\{111\}$ or $\{200\}$ ).

Measured pole figures are not normalised in multiples of a random pole figure.

Let $\hat{P}_{i}$ be the observed intensities of pole figure $h_{i}$. They must first be multiplied by an unknown normalisation coefficient $N_{i}$ before they can be compared to the recalculated pole figure $P_{h_{i}}(y)$, for which the following normalisation condition holds:

$$
\oint P_{h_{i}}(y) \mathrm{d} y=4 \pi
$$

It is hard to estimate $N_{i}$ for incomplete pole figures. Bunge (1969) proposed to treat them as additional unknowns in the minimization procedure of $\Delta$. Eq. (1) can also be written as:

$$
\Delta=\Sigma w_{i} \int_{B}\left[N_{i} \widehat{P}_{i}(y)-P_{h_{i}}(y)\right]^{2} \mathrm{~d} y=\operatorname{Min}
$$

In what we will subsequently call method $I$, the minimum of $\Delta$ is sought by setting its partial derivatives to $C_{l}^{\mu \nu}$ and $N_{i}$ equal to zero. This respectively leads to:

$$
\sum_{i} w_{i} \int_{B} \frac{\partial P_{h_{i}}(y)}{\partial C_{l}^{\mu \nu}}\left[N_{i} \hat{P}_{i}(y)-P_{h_{i}}(y)\right] \mathrm{d} y=0
$$

for all $l, \mu$ and $v$ except $l=0$, and:

$$
N_{i}=\int_{B} P_{h_{i}}(y) \hat{P}_{i}(y) \mathrm{d} y / \int_{B} \hat{P}_{i}^{2}(y) \mathrm{d} y
$$

for $i=1$ to $n$.

Note that, as can be derived from Eqs. (2-3),

$$
\frac{\partial P_{h_{i}}(y)}{\partial C_{l}^{\mu \nu}}=\frac{4 \pi}{2 l+1} \dot{k}_{l}^{\mu}\left(h_{i}\right) \dot{k}_{l}(y)
$$

Eqs. (6-7) lead to a set of linear equations in $C_{l}^{\mu \nu}$ and $N_{i}$ (Pospiech and Jura, 1974; Morris, 1975). 


\section{Method II}

Van Houtte (1980) also used criterion (5), however without treating the $N_{i}$ as unknowns during the minimization procedure.

He proposed the following expression for the $N_{i}$ :

$$
N_{i}=\int_{B} P_{h_{i}}(y) \mathrm{d} y / \int_{B} \hat{P}_{i}(y) \mathrm{d} y
$$

This resembles the classical expression for the normalisation of measured complete pole figures (Bunge, 1969):

$$
N_{i}=4 \pi / \oint \hat{P}_{i}(y) \mathrm{d} y
$$

It is seen that expresssion (9) is identical to expression (10) when $B=$ the complete pole figure. $N_{i}$ can then be calculated independently from the $C_{l}^{\mu \nu}$. This is not so for incomplete pole figures. In practice, Method II uses the same equations as Method I, except that Eq. (7) is replaced by Eq. (9). A linear set of equations in $C_{l}^{\mu \nu}$ and $N_{i}$ which can readily be solved is the result.

\section{THE NEW METHOD}

A new method will now be presented. It will be referred to as Method III. A close inspection of criterion (5) reveals, that the minimization is not applied to the squares of the differences between the experimental data and a "model" for them (i.e., the recalculated pole figures); but that the experimental data are first multiplied by a parameter which appears as an unknown during the minimization.

This is a quite unconventional data fitting procedure. A more conventional criterion is hereby proposed:

$$
\Delta_{E}=\sum_{i} w_{i} \int_{B}\left(\hat{P}_{i}-P_{h_{i}} / N_{i}\right)^{2} \mathrm{~d} y=\operatorname{Min}
$$

In order to avoid the predominance of the strongest pole figure, it is recommended that the weighing factors are chosen as follows:

$$
w_{i}=w_{i}^{\prime} / \int_{B} \hat{P}_{i}^{2} \mathrm{~d} y
$$

in which the $w_{i}^{\prime}$ can be set equal to 1 or can be given values that reflect the precision of each particular pole figure. 
The partial derivatives of $\Delta_{E}$ with respect to $C_{l}^{\mu \nu}$ and $N_{i}$ must be equal to zero. This respectively leads to the following conditions:

$$
\sum_{i} w_{i} \int_{B} \frac{\partial P_{h_{i}}(y)}{\partial C_{l}^{\mu \nu}}\left[\hat{P}_{i}(y)-P_{h_{i}}(y) / N_{i}\right] \mathrm{d} y=0
$$

for each combination of $l, \mu$ and $v$ except $l=0$, and

$$
N_{i}=\int_{B} P_{h_{i}}^{2}(y) \mathrm{d} y / \int_{B} \widehat{P}_{i}(y) P_{h_{i}}(y) \mathrm{d} y
$$

for $i=1$ to $n$.

Equations (13-14) form a non-linear set of equations which is very difficult to solve. However, there are nowadays other techniques available for finding the minimum of a function such as $\Delta_{E}$ (see appendix I).

\section{THEORETICAL ERROR ANALYSIS}

A short error analysis is presented here. It will make the comparison between the three methods easier.

Let the true texture function of a homogeneous sample be $f^{T}(g)$. The pole figures $P_{h_{i}}^{T}(y)$ are recalculated from it. $f^{T}$ and $P_{h_{i}}^{T}$ are usually unknown. $\hat{P}_{i}(y)$ are the measured incomplete pole figures. The experimental errors are defined by:

$$
\hat{\varepsilon}_{i}(y)=\hat{P}_{i}(y)-P_{h_{i}}^{T}(y) / N_{i}^{T}
$$

$N_{i}^{T}$ is a "true" normalisation factor.

A problem with $N_{i}^{T}$ and $\hat{\varepsilon}_{i}(y)$ is that in principle they are not unique. Indeed, suppose that both $\hat{P}_{i}(y)$ and $P_{h_{i}}^{T}(y)$ are known and that $N_{i}^{T}$ and $\hat{\varepsilon}_{i}(y)$ are to be calculated. It is seen from Eq. (15) that $N_{i}^{T}$ can freely be chosen and that the $\hat{\varepsilon}_{i}(y)$ depends on it. The lack of uniqueness only disappears when an additional assumption is made. Three examples of reasonable assumptions are:

$$
\int_{B} \hat{\varepsilon}_{i}^{2}(y) \mathrm{d} y=\operatorname{Min}
$$

or

$$
\int_{B} \hat{\varepsilon}_{i}(y) \mathrm{d} y=0
$$


or

$$
\int_{B}\left|\hat{\varepsilon}_{i}(y)\right| \mathrm{d} y=\operatorname{Min}
$$

There seems to be no way to tell which is the "right" assumption; a choice would be a convention. Note that the classical method for complete pole figures (Bunge, 1969) which uses Eq. (10) tacitly adopts the convention Eq. (17).

The differences between the true pole figures $P_{h_{i}}^{T}$ and the recalculated pole figures as found by the methods I, II and III are given by:

$$
\varepsilon_{i}^{T}(y)=P_{h_{i}}(y)-P_{h_{i}}^{T}(y)
$$

It is this error that really matters. A third kind of error is the "apparent experimental error" given by:

$$
\varepsilon_{i}(y)=\hat{P}_{i}(y)-P_{h_{i}}(y) / N_{i}
$$

in which $N_{i}$ is the normalisation factor as found by one of the three methods. It can be shown that

$$
\varepsilon_{i}^{T}(y)=N_{i}^{T} \hat{\varepsilon}_{i}(y)-N_{i} \varepsilon_{i}(y)+\left(N_{i}-N_{i}^{T}\right) \hat{P}_{i}(y)
$$

This equation is particularly instructive when a convention such as Eq. (16), (17) or (18) is adopted for the definition of $N_{i}^{T}$ and $\hat{\varepsilon}_{i}(y)$. The values of $\hat{\varepsilon}_{i}(y)$ are strongly restricted by doing so. Since the values of $\varepsilon_{i}(y)$ are restricted by the method used for the analysis of the pole figures, the last term of the sum in Eq. (21) becomes preponderant unless $N_{i}=$ $N_{i}^{T}$. It can thus be concluded that it is very important to obtain precise values for $N_{i}$ in order to avoid large errors of the type $\varepsilon_{i}^{T}(y)$.

\section{GENERATION OF ARTIFICIAL ERRORS IN POLE FIGURES RECALCULATED FROM KNOWN TEXTURE FUNCTIONS}

The usual test of a computer program for the analysis of incomplete pole figures consists of:

(i) - choosing a "true" texture function;

(ii) - calculation of several pole figures from it;

(iii) - treatment of these pole figures by the computer program as if they were incompletely measured pole figures. 
(iv)-comparing the resulting texture function with the original texture function.

In case series expansion methods are used, only the $C_{l}^{\mu v}$ coefficients of even rank $l$ are used for the comparison, the determination of the coefficients of odd rank $l$ requiring an additional treatment (e.g. Van Houtte, 1983).

The same scheme will be followed in the present work, except that artificial "experimental" errors will be introduced in the pole figures obtained in step (ii). Such tests have already been presented by Liang, $\mathrm{Xu}$ and Wang (1983).

\section{Choice of a "true" texture function}

The coefficients of even rank $l$ are chosen. The even part of the "true" texture function then is:

$$
\tilde{f}^{T}(g)=\sum_{l=0(2)}^{L T} \sum_{\mu=1}^{M(l)} \sum_{v=1}^{N(l)} C_{l}^{T \mu \nu} \dot{T}_{l}^{\mu v}(g)
$$

The coefficients of the series expansion of the recalculated "true" pole figures $P_{h_{i}}^{T}$ are given by the usual formula (Bunge, 1969):

$$
F_{l}^{T v}\left(h_{i}\right)=\frac{4 \pi}{2 l+1} \sum_{\mu=1}^{M(l)} C_{l}^{T \mu \nu} k_{l}^{\mu}\left(h_{i}\right)
$$

By convention, the value 1 is given to the theoretical normalisation factor $N_{i}^{T}$.

The artificial experimental pole figures are generated on a grid of pole figure directions $y_{j}$. We use a mesh length of $1^{\circ}$ in the $\beta$ direction and $(5 / 3)^{\circ}$ in the $\Phi$ direction:

$$
\hat{P}_{i}\left(y_{j}\right)=\left[1+\varepsilon_{1 i}\left(y_{j}\right)\right] P_{h_{i}}^{T}\left(y_{j}\right)+\varepsilon_{2 i}\left(y_{j}\right)+\varepsilon_{3 i}\left(y_{j}\right)
$$

The errors $\varepsilon_{1 i}$ and $\varepsilon_{2 i}$ are generated by:

$$
\varepsilon_{k i}(y)=\sum_{l=0(2)}^{L^{\varepsilon}}\left(E_{k_{i}}\right)_{l}^{v} \dot{k}_{l}^{v}(y)
$$

The coefficients $\left(E_{k_{i}}\right)_{l}^{v}$ are chosen independently for each pole figure as random numbers with a Gaussian distribution of which the averages are zero. The standard deviations $\sigma_{1}(l)$ and $\sigma_{2}(l)$ can be chosen at each test. Random numbers with a Gaussian distribution are used to generate the $\varepsilon_{3 i}$ on the grid positions $y_{j}$ (also independently for each pole figure). 
Their average is zero and their standard deviation $\sigma_{3}$. It is seen that $\varepsilon_{1 i}$ simulates possible errors on the unit intensity used to convert the measured intensities in relative intensities. $\varepsilon_{3 i}$ simulates high-frequency fluctuations, the highest possible frequencies being determined by the mesh length mentioned above.

Intensity fluctuations of lower frequencies can be simulated by $\varepsilon_{2 i}$. The choices of $\varepsilon_{1 i}, \varepsilon_{2 i}$ and $\varepsilon_{3 i}$ are made independently for different pole figures. Note that it has not been the intention here to simulate all possible types of experimental errors. The "experimental error" as defined by Eq. (15) finally would be:

$$
\hat{\varepsilon}_{i}\left(y_{j}\right)=\varepsilon_{1 i}\left(y_{j}\right) P_{h_{i}}^{T}\left(y_{j}\right)+\varepsilon_{2 i}\left(y_{j}\right)+\varepsilon_{3 i}\left(y_{j}\right)
$$

\section{QUALITY CRITERIA FOR THE RESULTS}

Let the maximal degree of the series expansion used by method I, II or III be $L$, and let the maximal degree of the series expansion required for a proper description of the "true" texture function (Eq. (22)) be $L^{T}$. The quality of the texture function $f(g)$ found by the methods could be evaluated by:

$$
\Delta_{f}=\oint\left[\tilde{f}(g)-\tilde{f}^{T}(g)\right]^{2} \mathrm{~d} g
$$

which should be minimal.

The following convention is now made:

$$
C_{l}^{T \mu \nu}=0 \quad \text { if } \quad l>L^{T}
$$

Equation (27) can be written as:

$$
\Delta_{f}=\sum_{l=0(2)}^{L} \sum_{\mu=1}^{M(l)} \sum_{\nu=1}^{N(l)} \frac{\left(C_{l}^{\mu \nu}-C_{l}^{T \mu \nu}\right)^{2}}{2 l+1}+\sum_{l=L+2(2)}^{\infty} \sum_{\mu=1}^{M(l)} \sum_{\nu=1}^{N(l)} \frac{\left(C_{l}^{T \mu \nu}\right)^{2}}{2 l+1}
$$

It is seen that the lowest possible value of $\Delta_{f}$ is obtained by the solution

$$
C_{l}^{\mu \nu}=C_{l}^{T \mu \nu} \text { for } l \leqslant L
$$

henceforward called "the ideal solution".

We now define

$$
\Delta_{C}(l)=\sum_{\lambda=0(2)}^{l} \sum_{\mu=1}^{M(\lambda)} \sum_{v=1}^{N(\lambda)} \frac{\left(C_{\lambda}^{\mu v}-C_{\lambda}^{T \mu \nu}\right)^{2}}{2 \lambda+1}
$$

$\Delta_{c}(L)$ is preferred to $\Delta_{f}$ as quality criterion for a solution, since the "ideal 
solution" not only minimizes it, but also makes it zero. Intermediate values $\Delta_{c}(l)$ (for $\left.l<L\right)$ are made zero as well.

\section{COMPLETE POLE FIGURES}

It is interesting to examine how the three methods behave when they are used to analyse complete pole figures.

TABLE I

Test cases for complete pole figures

\begin{tabular}{lcc}
\hline & $L \geqslant L^{T}$ & $L<L^{T}$ \\
\hline all $\left(\hat{E}_{i}\right)_{l}^{v}=0$ & A1 & A2 \\
$\left(\hat{E}_{i}\right)_{l}=0$ for $l \leqslant L$ & B1 & B2 \\
No restrictions & C1 & C2 \\
\hline
\end{tabular}

Let $\left(\hat{E}_{i}\right)_{l}^{v}$ be the coefficients of the series expansion of the experimental errors:

$$
\hat{\varepsilon}_{i}(y)=\sum_{l=0}^{\infty} \sum_{v=1}^{N(l)}\left(\hat{E}_{i}\right)_{l}^{v} \dot{k}_{l}^{v}(y)
$$

In Table I, six types of tests are summarized.

In cases $\mathrm{A} 1$ and $\mathrm{A} 2$ there are no experimental errors, while in cases B1 and B2 their series expansion only consists of terms of higher order than $L$.

The solution of one of the three methods (a set of $C_{l}^{\mu \nu}$ and $N_{i}$ ) must satisfy:

(i) Eq. (6) or Eq. (13) (partial derivative to $C_{l}^{\mu v}$ )

(ii) Eq. (7), Eq. (9) or Eq. (14) (partial derivatives to $N_{i}$ )

It will be shown that for complete pole figures, a solution consisting of the "ideal solution" of Eq. (30) with in addition

$$
N_{i}=N_{i}^{T}
$$

satisfies the conditions (i) for the test cases A1, A2, B1 and B2, while this is not so for the condition (ii). 


\section{Method I}

For complete pole figures, Eq. (6) can be transformed into:

$$
\sum_{i} w_{i} \dot{k}_{l}^{\mu}\left(h_{i}\right)\left[N_{i} \hat{F}_{l}^{v}\left(h_{i}\right)-F_{l}^{v}\left(h_{i}\right)\right]=0
$$

(for $l \leqslant L$ ). The coefficients $\hat{F}_{l}^{v}$ describe the observed pole figures.

This is satisfied by the ideal solutions Eq. (30) and Eq. (33) since in that case

$$
F_{l}^{v}\left(h_{i}\right)=F_{l}^{T v}\left(h_{i}\right)
$$

Besides, for the test cases A1, A2, B1 and B2

$$
\hat{F}_{l}^{v}\left(h_{i}\right)=F_{l}^{T v}\left(h_{i}\right) / N_{i}^{T}+\left(\hat{E}_{i}\right)_{l}^{v}
$$

and $\left(\hat{E}_{i}\right)_{l}^{v}=0$ for $l \leqslant L$.

On the other hand, Eq. (7) becomes:

$$
N_{i}=\frac{\sum_{l=0(2)}^{L} \sum_{v=1}^{N(l)} F_{l}^{v}\left(h_{i}\right) \hat{F}_{l}^{v}\left(h_{i}\right)}{\sum_{l=0(2)}^{L} \sum_{v=1}^{N(l)}\left[\hat{F}_{l}^{v}\left(h_{i}\right)\right]^{2}+\sum_{l=L+2(2)}^{\infty} \sum_{v=1}^{N(l)}\left[\hat{F}_{l}^{v}\left(h_{i}\right)\right]^{2}}
$$

It is seen that, regarding Eqs. (35-36), Eq. (37) is satisfied by the ideal solution if, and only if,

$$
\hat{F}_{l}^{v}\left(h_{i}\right)=0 \text { for } l>L
$$

This is only so in case A1, not in cases A2, B1, B2. It can be concluded that Method I can only find the "ideal" solution in case A1 (Table I).

\section{Method II}

Equation (6) is satisfied in the same way as for method I.

Equation (9) becomes:

$$
N_{i}=F_{0}^{1} / \hat{F}_{0}^{1}
$$

This is equivalent to Eq. (33) as long $\left(\hat{E}_{i}\right)_{0}^{1}=0$. So method II finds the ideal solution in test cases A1, A2, B1 and B2. 


\section{Method III}

Equation (13) becomes:

$$
\sum_{i} w_{i} \dot{k}_{l}^{\mu}\left(h_{i}\right)\left[\hat{F}_{l}^{v}\left(h_{i}\right)-F_{l}^{v}\left(h_{i}\right) / N_{i}\right]=0
$$

(for $l \leqslant L$ )

which is satisfied by the ideal solution in the test cases A1, A2, B1 and B2 in the same way as Eq. (34).

Equation (14) becomes:

$$
N_{i}=\frac{\sum_{l=0(2)}^{L}\left[F_{l}^{v}\left(h_{i}\right)\right]^{2}}{\sum_{l=0(2)}^{L} \hat{F}_{l}^{v}\left(h_{i}\right) F_{l}^{v}\left(h_{i}\right)}
$$

which is equivalent to Eq. (33) if $\hat{F}_{l}^{v}=F_{l}^{T^{v}}$ for $l \leqslant L$. So method III also finds the ideal solution in test cases A1, A2, B1 and B2.

Neither of the three methods will probably find the ideal solution in the test cases $\mathrm{C} 1$ and $\mathrm{C} 2$.

\section{COMPARISON OF THE THREE METHODS BY MEANS OF COMPUTER SIMULATIONS}

Computer simulation still allow the comparison when analytical methods fail. Incomplete pole figures containing artificial errors were prepared to be analyzed by the three methods.

Two textures were chosen as "true textures", a rather sharp one $\left(L^{T}=34\right)$ and one of medium sharpness $\left(L^{T}=22\right)$.

\section{Sharp texture}

The sharp texture is a classical cold rolling texture of f.c.c. metals as found by the Taylor theory (Van Houtte, 1978). A rolling reduction of $86 \%$ was simulated on 147 crystallites. The resulting set of discrete orientations was transformed into a continuous orientation distribution function by putting a Gauss distribution upon each of the orientations. The spread of the Gaussians is characterised by $\Phi_{0}=7^{\circ}$ (see Eq. (6.45), Bunge, 1969). A series expansion up to $l=34$ is sufficient for the description of this texture which has a texture index of 11.93. Figure 1 shows th ecalculated $\{111\}$ and $\{100\}$ pole figures. 


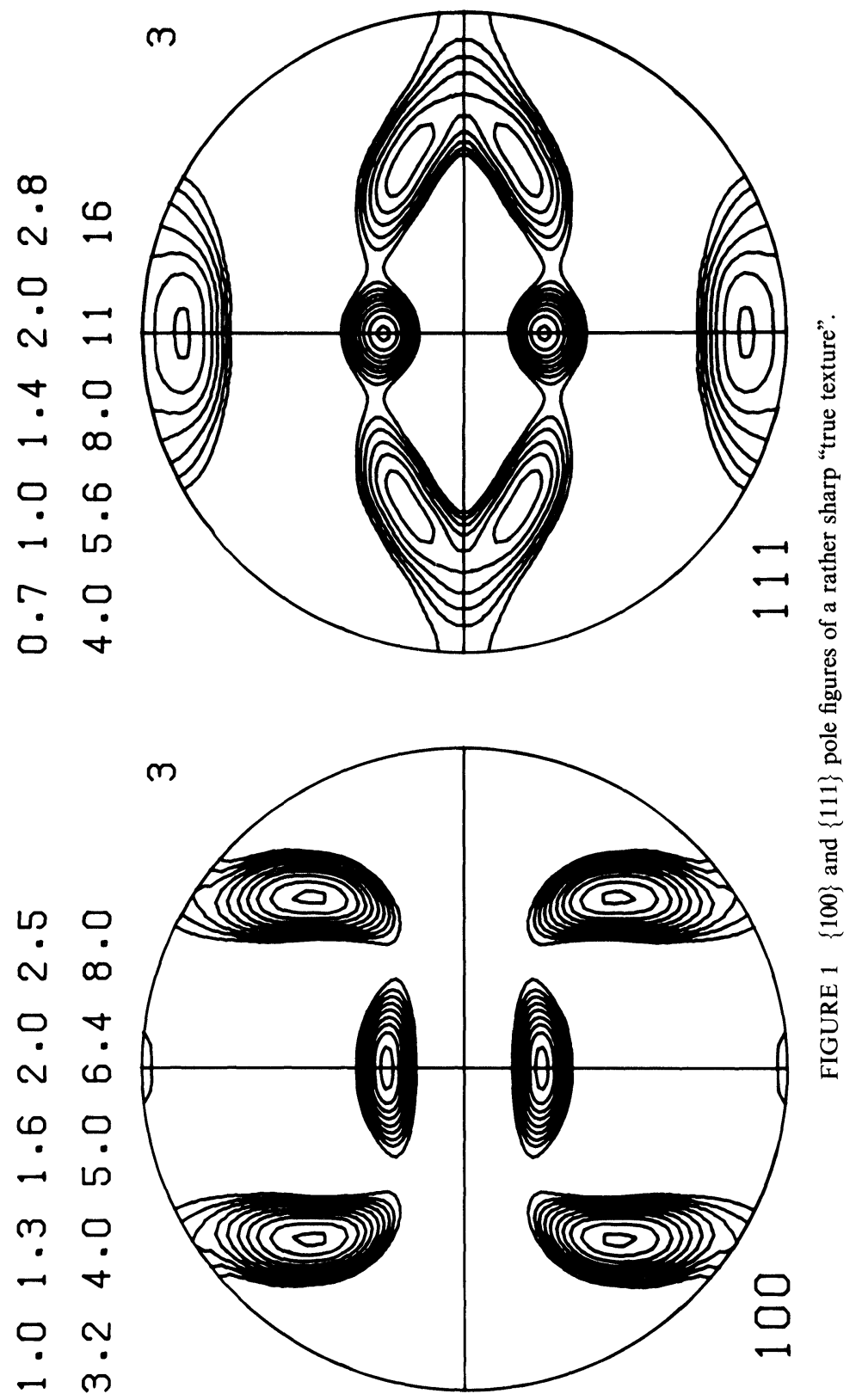


The $\{110\}$ and $\{311\}$ pole figures were recalculated as well, so that 4 pole figures were available to construct artificial "experimental pole figures". All tests were performed on 4 pole figures (complete or incomplete). A first series of tests was designed to investigate the influence of $L / L^{T}$. No experimental errors were generated.

Table II shows the results for $\Phi_{m}=80^{\circ}$ (case of incomplete pole figures). The values found for the normalisation factors and the quality criterion $\Delta_{c}(l)$ (Eq. (31)) are used for the comparison of the methods.

It can theoretically be proved, that for $L=34$, the three methods find the "ideal solution" described by Eqs. $(30,33)$.

At $L=22\left(L / L^{T}=0.65\right), \Delta_{c}(22)$ indicates a comparable quality for method I and II, and a better one for method III. Method I slightly underestimates the normalisation factors by max. $1.6 \%$, while methods II and III identify them with a precision better than $0.3 \%$.

At $L=16\left(L / L^{T}=0.47\right), \Delta_{c}(16)$ indicates a much worse result for Method I than for the two other methods, III being the best. Method I underestimates the normalisation factors by max. $11 \%$; methods I and II reach a precision better than $0.5 \%$.

A test has also been carried out for complete pole figures and $L=16$ (Table III). It was shown above that method II and III should find the ideal solution in that case. The value 0.0009 found by method III for $\Delta_{c}(16)$ can be ascribed to errors in the numerical integrations. Method I still underestimates the normalisation factors by a maximum of $10 \%$. Artificial experimental errors of the type $\varepsilon_{1}$ and $\varepsilon_{2}$ (Eq. (24)) were introduced in a second series of tests. The parameters used for the generation of them are given by Table IV. Figure 2 shows the resulting $\{111\}$ and $\{100\}$ pole figures.

TABLE II

Comparison by computer simulation

\begin{tabular}{|c|c|c|c|c|c|c|c|}
\hline \multirow[b]{2}{*}{ Method: } & \multicolumn{3}{|c|}{$L=22$} & \multicolumn{3}{|c|}{$L=16$} & \multirow{2}{*}{$\begin{array}{c}\text { "True Value" } \\
N_{i}^{T}\end{array}$} \\
\hline & I & II & III & I & II & III & \\
\hline$N_{1}(100)$ & 0.984 & 1.000 & 0.998 & 0.892 & 1.001 & 0.996 & 1 \\
\hline$N_{2}(110)$ & 0.989 & 0.996 & 1.001 & 0.901 & 1.000 & 1.002 & 1 \\
\hline$N_{3}(111)$ & 0.989 & 1.002 & 1.002 & 0.900 & 1.003 & 1.003 & 1 \\
\hline$N_{4}(311)$ & 0.996 & 1.000 & 1.001 & 0.954 & 1.000 & 1.004 & 1 \\
\hline$\Delta_{c}(16)$ & 0.0056 & 0.0021 & 0.0017 & 0.1436 & 0.0490 & 0.0356 & \\
\hline$\Delta_{C}(22)$ & 0.0720 & 0.0705 & 0.0273 & & & & \\
\hline
\end{tabular}

True texture: (Figure 1) $L^{T}=34 \quad$ Texture index $=11.93$

Artificial errors: None

Incomplete pole figures: $\left(\Phi_{m}=80^{\circ}\right)$ 
TABLE III

Comparison by computer simulation

\begin{tabular}{lccc}
\hline & \multicolumn{2}{c}{$L=16$} & True value \\
\cline { 1 - 3 } Method: & I & III & $N_{i}^{T}$ \\
\hline$N_{1}(100)$ & 0.9034 & 1.0000 & 1 \\
$N_{2}(110)$ & 0.9029 & 1.0000 & 1 \\
$N_{3}(111)$ & 0.9015 & 1.0000 & 1 \\
$N_{4}(311)$ & 0.9526 & 1.0000 & 1 \\
\hline$\Delta_{c}(16)$ & 0.0996 & 0.0009 & \\
\hline
\end{tabular}

True texture: (Figure 1) $L^{T}=34 \quad$ Texture index $=11.93$

Artificial errors: none

Complete pole figures: $\left(\Phi_{m}=90^{\circ}\right)$

\section{TABLE IV}

Choice of the artificial errors as defined by Eqs. $(24-25)$

$\varepsilon_{1 i}:\left\{\begin{array}{l}\sigma_{1}(l)=0.04 \text { for } 0 \leqslant l \leqslant 16 \\ \sigma_{1}(l)=0 \text { for } l>16\end{array}\right.$
$\varepsilon_{2 i}=0$ or $\sigma_{2}(l)=0$ for all $l$
$\varepsilon_{3 i}: \sigma_{3}=0.2$

Table V gives the results for $\Phi_{m}=80^{\circ}$. There is no significant difference between the three methods for $L=34\left(L / L^{T}=1\right)$. The estimation of the normalisation factors by method I are approximately $3 \%$ lower than those obtained by the two other methods at $L=22\left(L / L^{T}=0.65\right)$. According to $\Delta_{c}$ (22), method III is then better than method I and II. At $L=16\left(L / L^{T}=0.47\right)$, methods II and III are much better than method I, which gives approximately $13 \%$ lower values for the normalisation coefficients.

The last column of Table $\mathrm{V}$ gives the normalisation coefficients which were obtained from the complete pole figures by the classical method (Eq. (10)).

\section{TEXTURE OF MEDIUM SHARPNESS}

A less sharp texture has also been chosen for the tests. It is the result of the "lath model", a variant of the Taylor theory valid for metals with elongated grains (Van Houtte, 1982). A rolling reduction of $86 \%$ of a 
DETERMINATION OF TEXTURE FUNCTIONS

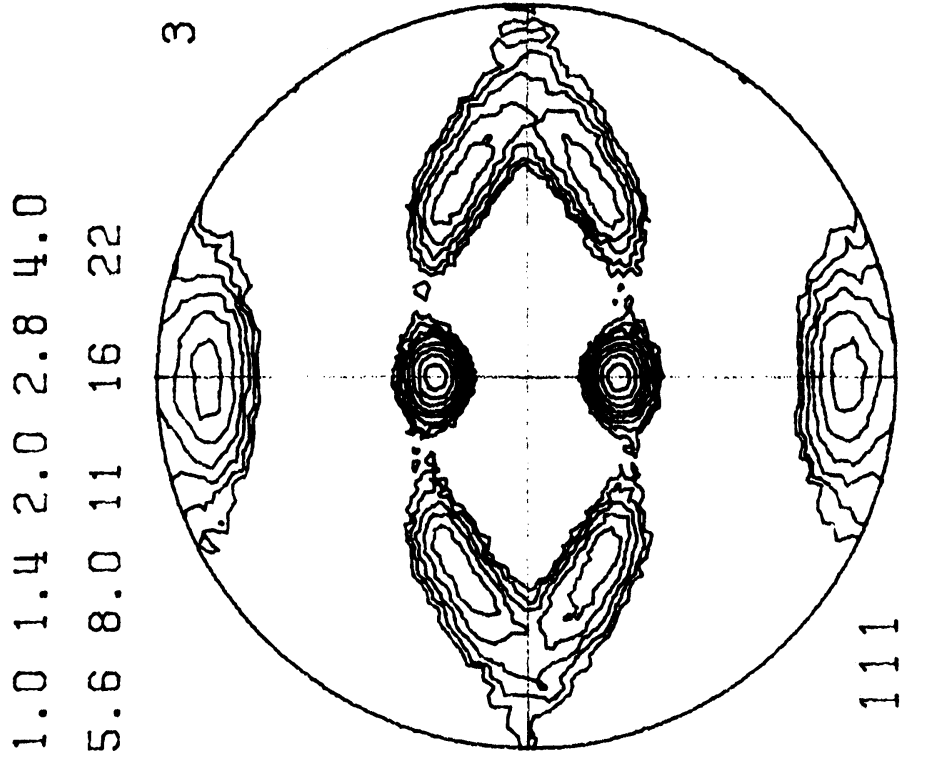

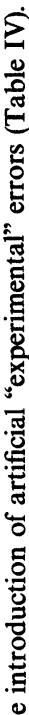

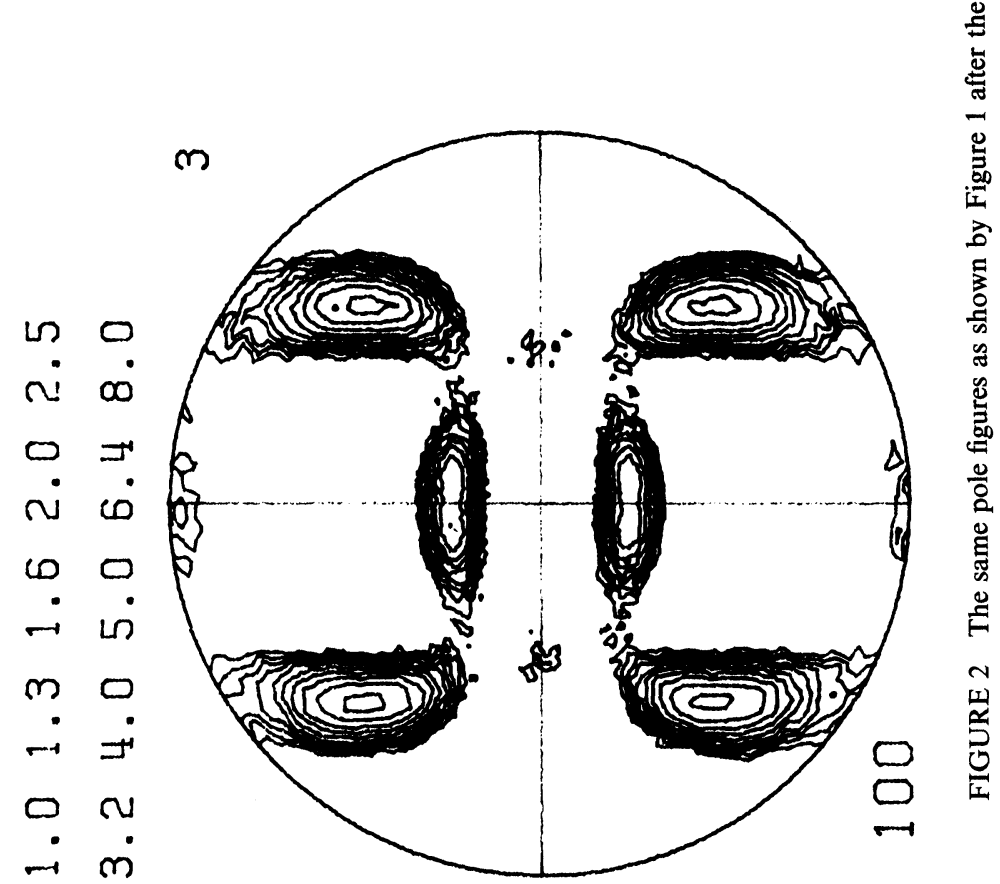




\section{TABLE V}

Comparison by computer simulation

\begin{tabular}{|c|c|c|c|c|c|c|c|c|c|c|c|}
\hline \multirow[b]{2}{*}{ Method: } & \multicolumn{3}{|c|}{$L=34$} & \multicolumn{3}{|c|}{$L=22$} & \multicolumn{3}{|c|}{$L=16$} & \multirow{2}{*}{$\begin{array}{c}\text { True } \\
\text { value } \\
N_{i}^{T}\end{array}$} & \multirow{2}{*}{$\begin{array}{c}N_{i} \\
\text { from } \\
\text { Eq. }(10)\end{array}$} \\
\hline & I & II & III & I & II & III & I & II & III & & \\
\hline$N_{1}(100)$ & 0.967 & 0.987 & 0.991 & 0.953 & 0.989 & 0.989 & 0.860 & 0.990 & 0.987 & 1 & 0.986 \\
\hline$N_{2}(110)$ & 1.001 & 1.010 & 1.024 & 0.989 & 1.009 & 1.025 & 0.896 & 1.010 & 1.023 & 1 & 1.005 \\
\hline$N_{3}(111)$ & 0.930 & 0.952 & 0.951 & 0.918 & 0.956 & 0.954 & 0.825 & 0.956 & 0.956 & 1 & 0.973 \\
\hline $\mathrm{N}_{4}(311)$ & 0.959 & 0.988 & 0,980 & 0.955 & 0.988 & 0.981 & 0.912 & 0.989 & 0.984 & 1 & 0.999 \\
\hline$\overline{\Delta_{c}(16)}$ & 0.044 & 0.039 & 0.037 & 0.049 & 0.036 & 0.035 & 0.222 & 0.079 & 0.067 & & \\
\hline$\Delta_{c}(22)$ & 0.071 & 0.067 & 0.064 & 0.120 & 0.114 & 0.074 & & & & & \\
\hline$\Delta_{c}(34)$ & 0.167 & 0.164 & 0.157 & & & & & & & & \\
\hline
\end{tabular}

True texture: (Figure 1) $L^{T}=34 \quad$ Texture index $=11.93$

Artificial errors: Table IV-Figure 2

Incomplete pole figures: $\left(\Phi_{m}=80^{\circ}\right)$

The last column gives the normalisation factors which would be obtained by the classical method for complete pole figures.

f.c.c. metal was simulated. The resulting set of 294 crystallite orientations was transformed into a continuous ODF by putting a Gaussian distribution of $\Phi_{c}=10^{\circ}$ upon each of them. This texture has a texture index of $3.65 ; L^{T}=22$. Figure 3 shows the $\{100\}$ and $\{111\}$ pole figures. Several test series have been carried out for different types of artificial experimental errors.

Four incomplete pole figures were each time analysed $(\{100\},\{110\}$, $\{111\}$ and $\{311\}) ; L$ was chosen as 16 or 22 .

No significant differences between the results of the methods I, II or III have been found except in a case with particularly large artificial errors (Table VI and VII). Figure 4 shows two of the four artificial pole figures.

\section{TABLE VI}

Choice of artificial errors (as defined by Eqs.

(24-25))

\begin{tabular}{|c|c|}
\hline $\begin{array}{l}\varepsilon_{1 i}=0 \text { or } \sigma_{1}(l)=0 \text { for all } l \\
\varepsilon_{2 i:}: \begin{array}{l}\sigma_{2}(l)=0 \text { for } 0 \leqslant l \leqslant 22 \text { and } \\
\sigma_{2}(l)=0.1 \text { for } 22<l \leqslant 34\end{array}\end{array}$ & $l>34$ \\
\hline$\varepsilon_{3 i}=0$ for all $y_{i}$ & \\
\hline
\end{tabular}




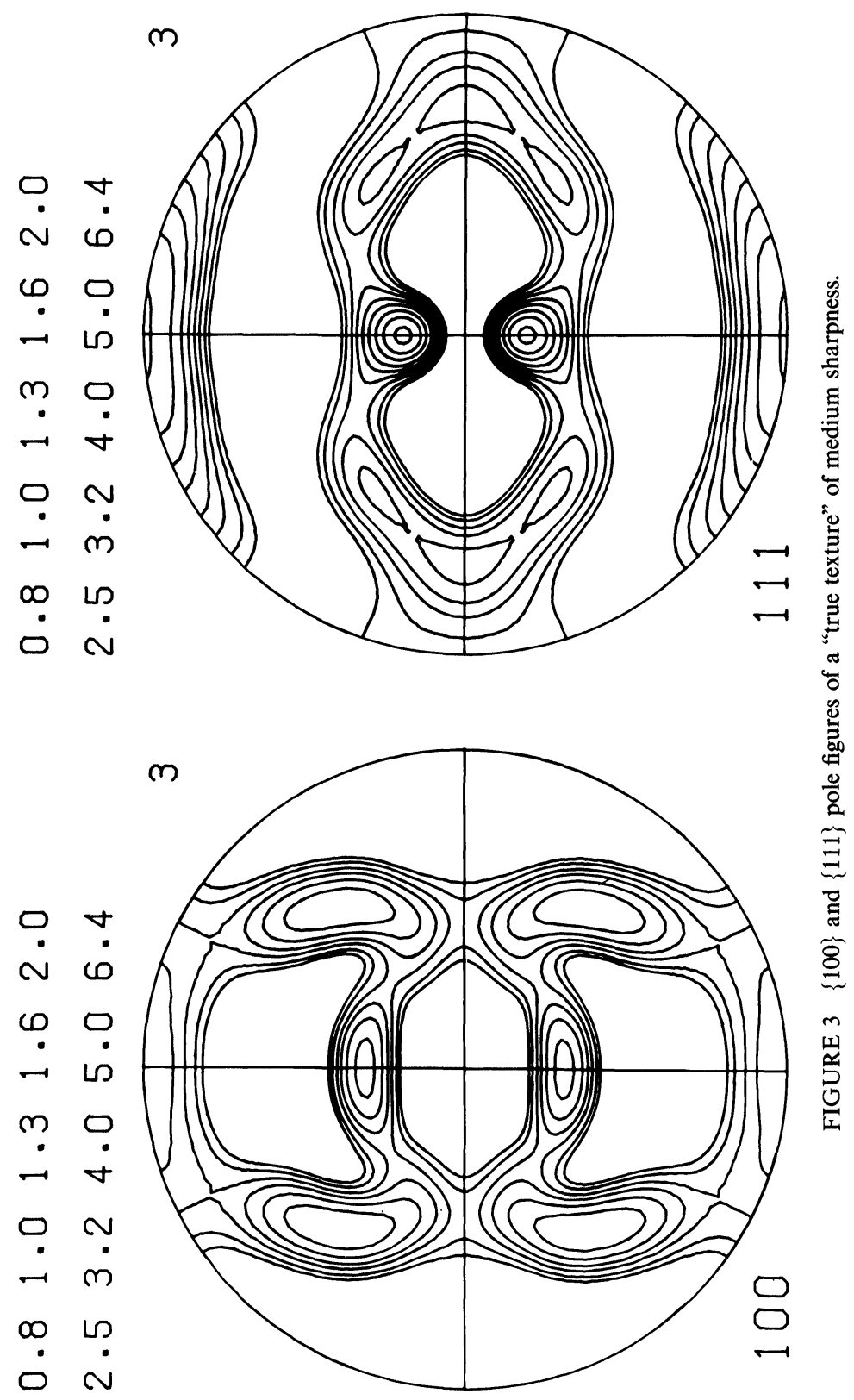




\section{TABLE VII}

Comparison by computer simulation

\begin{tabular}{|c|c|c|c|c|}
\hline & $L=22$ & Tru & & True \\
\hline Method: & I & II & III & $N_{i}^{T}$ \\
\hline$N_{1}(100)$ & 0.943 & 1.001 & 0.999 & 1 \\
\hline$N_{2}(110)$ & 0.934 & 1.003 & 1.002 & 1 \\
\hline$N_{3}(111)$ & 0.942 & 1.001 & 1.003 & 1 \\
\hline$N_{4}(311)$ & 0.934 & 0.999 & 1.001 & 1 \\
\hline$\Delta_{c}(16)$ & 0.014 & 0.005 & 0.004 & \\
\hline$\Delta_{c}(22)$ & 0.066 & 0.063 & 0.053 & \\
\hline
\end{tabular}

True texture: (Figure 3) $L^{T}=22 \quad$ Texture index $=3.65$

Artificial errors: Table VI-Figure 4

Incomplete pole figures: $\Phi_{m}=80^{\circ}$

\section{EXAMPLE FOR AN EXPERIMENTAL TEXTURE}

A $99.5 \%$ pure aluminium sheet was $90 \%$ cold rolled. The $\{111\},\{200\}$, $\{220\}$ and $\{311\}$ pole figures were incompletely measured at the surface by the back reflection method $\left(\Phi_{m}=80^{\circ}\right)$. They have been analysed by method I and method III with $L=34$ in both cases.

Table VIII shows the resulting normalisation factors.

TABLE VIII

Normalisation factors found for a cold rolled aluminium sample by method I and III $(L=34)$. Incomplete pole figures: $\Phi_{m}=80^{\circ}$

\begin{tabular}{lcc}
\hline & \multicolumn{2}{c}{$L=34$} \\
\hline Method: & I & III \\
\hline$N_{1}(100)$ & 0.604 & 0.700 \\
$N_{2}(110)$ & 0.613 & 0.705 \\
$N_{3}(111)$ & 0.621 & 0.755 \\
$N_{4}(311)$ & 0.731 & 0.825 \\
\hline
\end{tabular}

It is seen that the normalisation factors found by method I are up to $18 \%$ lower than those found by method III.

The $C_{l}^{\mu \nu}$ coefficients for which $l \leqslant 22$ were used as input data for the quadratic method. This method permits the calculation of a completely non-negative texture function, the even part of which comes as close as possible to the input data. It is one of the possible methods for finding 


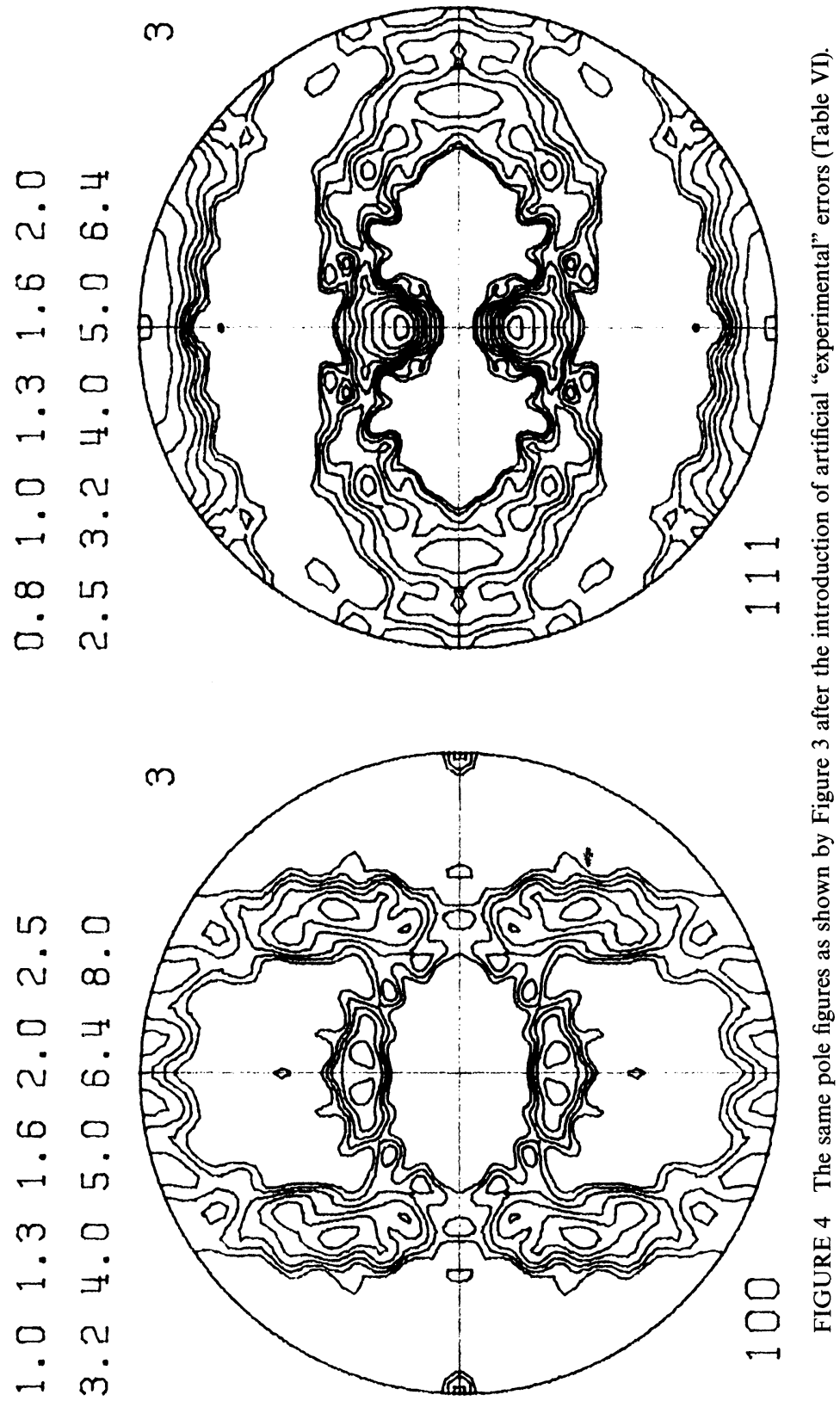


the odd part of a texture function, which can not be directly determined from measured pole figures (Van Houtte, 1983). It is preferred to limit the series expansions to $l=22$ in this method because of the considerable computer time involved. Since the resulting texture function is the square of a series expansion for $l$ up to 22 , it is nevertheless capable of a satisfactory representation of rather sharp textures.

Figures 5-6 show the resulting non-negative texture functions. The result of method III is sharper than that of method I (a maximum of 50.3 vs. 43.6). Both texture functions contain some small false peaks, which are no "ghosts" in the usual sense since the odd part of the texture function is not missing, but are to be ascribed to the lack of compatibility between the experimental pole figures. This lack of compatibility, normally due to experimental errors, is probably enhanced when inaccurate values of the normalisation factors are used.

It is seen that the false peaks are much more important for the result of method I than for the result of method III.

\section{CONCLUSIONS}

The theoretical error analysis shows that it is very important to obtain accurate values for the normalisation factors when analysing incomplete pole figures.

The analysis of simulated incomplete pole figures containing errors did not indicate significant differences between the three methods in the case of weak original textures. However, such differences do exist for sharp textures, especially when $L^{T}>L$, i.e. the original textures are so sharp that a series expansion of a higher rank than the one used by the method of the analysis is required for a proper description. All three methods have trouble in finding a satisfactory texture function in such cases. However, as can be seen in Tables II and V, the results of method I are much poorer than those of the two other methods. Most striking is the significant underestimation of the normalisation factors. This is even true when the pole figures to be analysed have no errors (Table II), or are complete, or both (Table III).

The underestimation of the normalisation coefficients by method I can be expected to be of the order of $10 \ldots 20 \%$ when $L / L^{T} \approx 0.5$. A practical example for a cold rolled aluminium sample shows that such sharp textures are by no means exceptional, even when the analysis is carried out with $L=34$. 


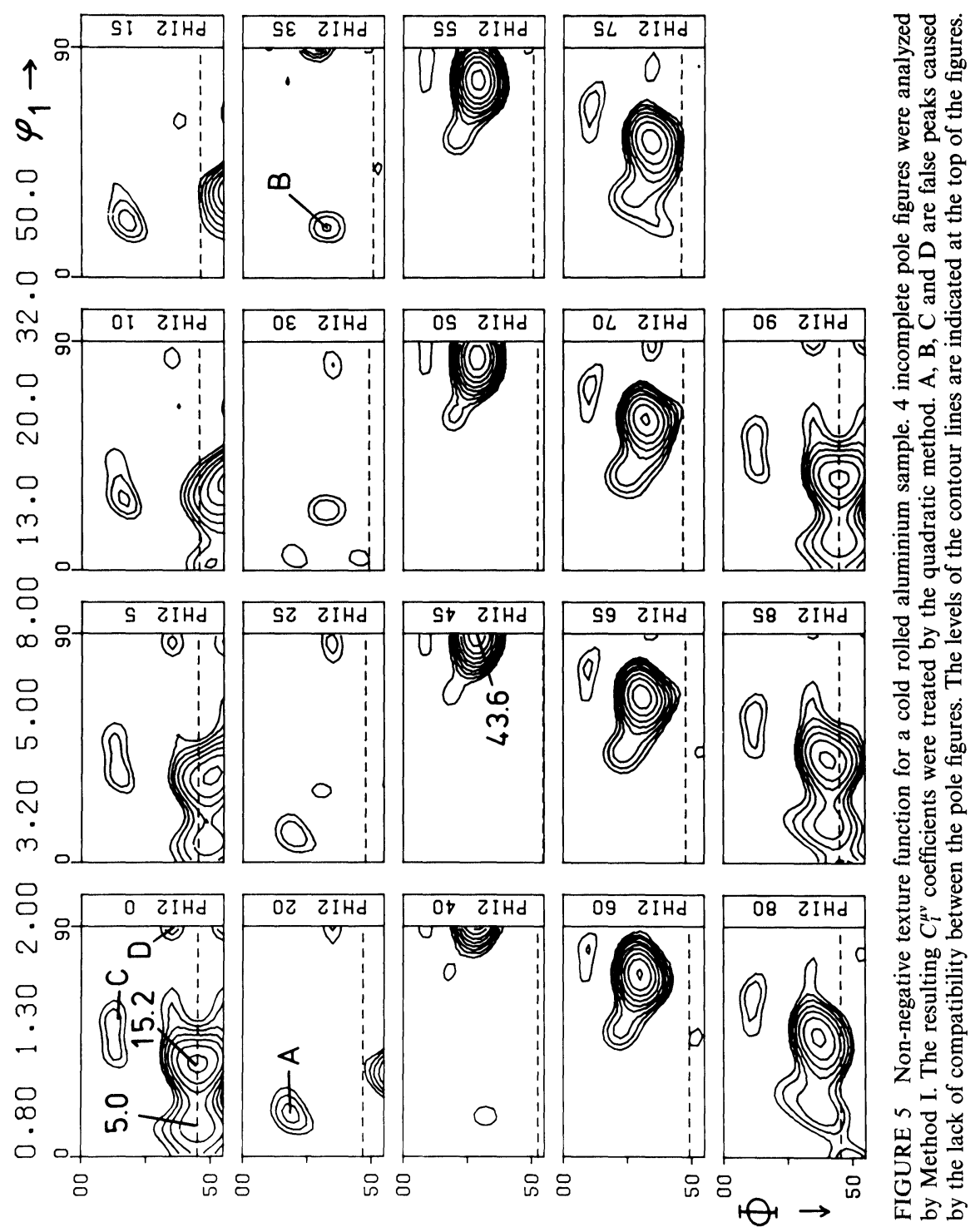




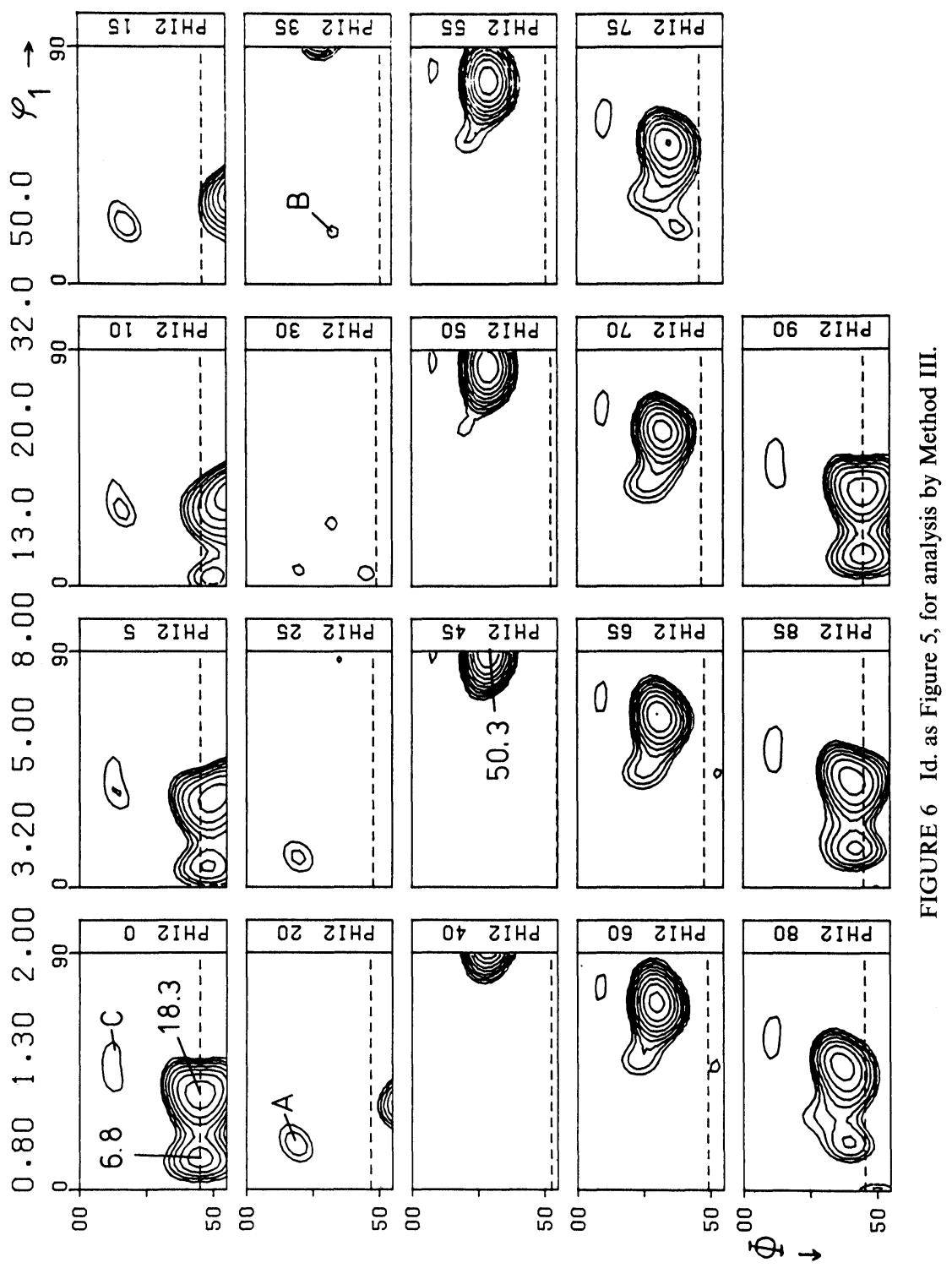


The two other methods give much more accurate results for the normalisation factors, at least when compared to the values which the classical method for complete pole figures would find (Table V). Method II gives the best normalisation coefficient while on the whole, the best results are obtained by method III.

Another interesting conclusion is that for sharp textures, it is always advantageous to choose $L$ as high as possible, even if one is not interested in $C_{l}^{\mu v}$ coefficients of such high rank $l$.

Suppose that only the coefficients for $l$ up to 16 are really needed.

It can be seen in Table II and V that $\Delta_{c}(16)$ is much smaller and hence better for $L=34$ or 22 than for $L=16$, regardless of the method which was used for the analysis. The most spectacular loss in quality is observed for method I.

\section{References}

Bunge, H. J. Z. Metallkd. 56, 872-874 (1956).

Bunge, H. J. Mathematische Methoden der Texturanalyse, Akademie-Verlag: Berlin (DDR) (1969).

Fletcher, R. Practical Methods of Optimization, vol. 1, John Wiley \& Sons, Chichester-New York-Brisbane-Toronto: (1980).

Humbert, M. and Bergmann, H. W. J. Appl. Cryst. 13, 500-504 (1980).

Imhof, J. Textures and Microstructures 5, 73-86 (1982).

Liang, Z., Xu, J. and Wang, F. Proc. Sixth International Conference on Textures of Materials, pp. 1259-1264. The Iron and Steel Institute of Japan. Tokyo (1981).

Liang, Z., Xu, J. and Wang, F. Mat. Sci. Eng. 60, 59-63 (1983).

Morris, P. R. Adv. X-ray Anal. 18, 515-534 (1975).

Pospiech, J. and Jura, J. Z. Metallkde 27, 324-330 (1974).

Roe, R. J. J. Appl. Phys. 36, 2024-2031 (1965).

Ruer, D. and Baro, R. J. Appl. Cryst. 10, 458-464 (1977).

Van Houtte, P. Acta Metall. 26, 591-604 (1978).

Van Houtte, P. Mat. Sci. Eng. 43, 7-11 (1980).

Van Houtte, P. Proc. Sixth International Conference on Textures of Materials, pp. 428437. The Iron and Steel Institute of Japan. Tokyo (1981).

Van Houtte, P. Textures and Microstructures 6, 1-20 (1983).

Williams, R. D. Trans. TMS-AIME 242, 105-115 (1968).

\section{APPENDIX I. SOLUTION OF METHOD III}

The problem is to find a set of $C_{l}^{\mu v}$ and $N_{i}$ which minimizes $\Delta_{E}$ as given by Eq. (11).

Setting the partial derivatives of $\Delta_{E}$ equal to zero leads to a set of non-linear equations (Eqs. (13-14)) which is very hard to solve. Fletcher (1980) mentions several alternative methods for the solution of such a problem, among which Newton's method which will be used here. 
First of all, we will replace the variables $N_{i}$ by variables $M_{i}$ :

$$
M_{i}=1 / N_{i}
$$

Eq. (11) then becomes an expression of the fourth order in the unknowns $C_{l}^{\mu v}$ and $M_{i}$.

$$
\Delta_{E}=\sum_{i=1}^{n} w_{i} \int_{B}\left[\hat{P}_{i}(y)-M_{i} P_{h_{i}}(y)\right]^{2} \mathrm{~d} y=\operatorname{Min}
$$

in which $P_{h_{i}}(y)$ is a series expansion (eq. (2)) of which the coefficients $F_{l}^{v}\left(h_{i}\right)$ are themselves linear functions of $C_{l}^{\mu \nu}$ (Eq. (3)).

In practice, we must take into account that

$$
C_{0}^{11}=1
$$

This simply means that partial derivatives to $C_{0}^{11}$ need not be considered.

An approximate solution is first obtained by method II. Let the result be $C_{0 l}^{\mu \nu}$ and $M_{0 i}$.

The fourth-order expression Eq. (43) is now replaced by a secondorder expression $\Delta_{E}^{\prime}$ which is in fact its Taylor expansion around $\left(C_{0 l}^{\mu \nu}, M_{0 i}\right)$. The minimum of the second order expression $\Delta_{E}^{\prime}$ is an estimation of the minimum of $\Delta_{E}$. It can be found in the classical way:

$$
\begin{aligned}
\frac{\partial \Delta_{E}^{\prime}}{\partial C_{l}^{\mu \nu}}=\frac{\partial \Delta_{E}(0)}{\partial C_{l}^{\mu \nu}}+\sum_{l^{\prime}} \sum_{\mu^{\prime}} \sum_{v^{\prime}} \frac{\partial^{2} \Delta_{E}(0)}{\partial C_{l}^{\mu \nu} \partial C_{l}^{\mu^{\prime} v^{\prime}}}\left(C_{l^{\prime}}^{\mu^{\prime} v^{\prime}}-C_{0 l^{\prime}}^{\mu^{\prime} v^{\prime}}\right) \\
+\sum_{j} \frac{\partial^{2} \Delta_{E}(0)}{\partial C_{l}^{\mu \nu} \partial M_{j}}\left(M_{j}-M_{0 j}\right)=0
\end{aligned}
$$

and

$$
\begin{aligned}
\frac{\partial \Delta_{E}^{\prime}}{\partial M_{i}}=\frac{\partial \Delta_{E}(0)}{\partial M_{i}}+\sum_{l^{\prime}} \sum_{\mu^{\prime}} \sum_{v^{\prime}} \frac{\partial^{2} \Delta_{E}(0)}{\partial M_{i} \partial C_{l}^{\mu^{\prime} v^{\prime}}}\left(C_{l^{\prime}}^{\mu^{\prime} v^{\prime}}-C_{0 l^{\prime}}^{\mu^{\prime} v^{\prime}}\right) & \\
& +\sum_{j} \frac{\partial^{2} \Delta_{E}(0)}{\partial M_{i} \partial M_{j}}\left(M_{j}-M_{0 j}\right)=0
\end{aligned}
$$

in which expressions such as $\partial \Delta_{E}(0) / \partial M_{i}$ stand for the numerical value of the corresponding partial derivative of $\Delta_{E}$ at the approximate solution $\left(C_{0 l}^{\mu \nu}, M_{0 i}\right)$. They can all be expressed analytically. For example

$$
\frac{\partial^{2} \Delta_{E}(0)}{\partial C_{l}^{\mu \nu} \partial C_{l^{\prime}}^{\mu^{\prime} v^{\prime}}}=\sum_{i} \frac{2 w_{i} M_{0 i}^{2}(4 \pi)^{2} \dot{k}_{l}^{\mu}\left(h_{i}\right) k_{l^{\prime}}^{\mu^{\prime}}\left(h_{i}\right) \zeta_{l l^{\prime}}^{v v^{\prime}}}{(2 l+1)\left(2 l^{\prime}+1\right)}
$$


For $\zeta_{u^{\prime}}^{v v^{\prime}}$, see Eq. (4.171) in the work of Bunge (1969). Expression (47) is zero if $v \neq v^{\prime}$.

Eqs. (45-46) form a set of linear equations in $C_{l}^{\mu v}$ and $M_{i}$. Its coefficient matrix contains many zeroes (see Eq. (47)) which, when properly exploited, greatly reduces the CPU time required for its solution. The solution is then used as a new approximate solution $\left(C_{0 l}^{\mu \nu}, M_{0 i}\right)$ and the procedure is repeated.

Table IX gives typical values for the correction on $M_{0 i}$ at successive iteration steps.

TABLE IX

Evaluation of the correction on

\begin{tabular}{cc}
\multicolumn{2}{c}{$M_{0 i}$ for $M_{0 i} \approx 1$} \\
\hline Step & $M_{i}-M_{0 i}$ \\
\hline 1 & $5 \times 10^{-3}$ \\
2 & $5 \times 10^{-5}$ \\
3 & $5 \times 10^{-7}$ \\
4 & $10^{-7}$ \\
\hline
\end{tabular}

For all tests which have been carried out until now, three iteration steps were sufficient to find the minimum of $\Delta_{E}$ with great accuracy. 